MATHEMATICS OF COMPUTATION

Volume 70, Number 236, Pages 1503-1514

S 0025-5718(00)01257-6

Article electronically published on March 30, 2000

\title{
THE NUMERICAL SOLUTION \\ OF INTEGRAL-ALGEBRAIC EQUATIONS OF INDEX 1 BY POLYNOMIAL SPLINE COLLOCATION METHODS
}

\author{
J.-P. KAUTHEN
}

\begin{abstract}
In this paper, we study polynomial spline collocation methods applied to a particular class of integral-algebraic equations of Volterra type. We analyse mixed systems of second and first kind integral equations. Global convergence and local superconvergence results are established.
\end{abstract}

\section{INTRODUCTION}

In this paper, we study numerical methods for the solution of a mixed system of Volterra integral equations of the first and second kind. More precisely, we consider the system

$$
\begin{aligned}
y(t) & =f(t)+\int_{0}^{t}\left(K_{11}(t, s) y(s)+K_{12}(t, s) z(s)\right) d s, \\
0 & =g(t)+\int_{0}^{t}\left(K_{21}(t, s) y(s)+K_{22}(t, s) z(s)\right) d s,
\end{aligned}
$$

and its nonlinear counterpart

$$
\begin{aligned}
y(t) & =f(t)+\int_{0}^{t} k(t, s, y(s), z(s)) d s, \\
0 & =g(t)+\int_{0}^{t} \ell(t, s, y(s), z(s)) d s .
\end{aligned}
$$

Here we assume that $t \in I=[0, T]$ and that the data functions $f, g, K_{i j}, i, j=1,2$, $k$ and $\ell$ are sufficiently smooth. Furthermore we suppose that $g(0)=0,\left|K_{22}(t, t)\right| \geq$ $k_{0}>0$ for all $t \in I$ and that the partial derivative of the function $\ell$ w.r. to its fourth variable $\partial_{4} \ell(t, t, y, z)$ is invertible for $t \in I$ and $y, z$ in a neighborhood of the solution. It then follows that the systems (1.1), (1.2) or (1.3), (1.4), respectively, have a continuous solution $y, z$ on $I$. This can be seen easily as follows. We first differentiate equations (1.2) and (1.4) w.r. to $t$ and consider the resulting equations as equations of the second kind for $z$. We formally solve for $z$ and replace the resulting expressions in (1.1), (1.3), respectively. We thus obtain equations of the second kind for $y$. However it has to be pointed out that this reduction to an integral equation of the second kind for $y$ is not practical from a numerical point of view.

Received by the editor April 1, 1997 and, in revised form, September 8, 1999.

2000 Mathematics Subject Classification. Primary 65R20.

Key words and phrases. Volterra equation, integral-algebraic, spline collocation method.

(C)2000 American Mathematical Society 
Coupled systems of Volterra equations of the first and second kind arise for example in a slightly different form in problems of identification of memory kernels in heat conduction and viscoelasticity (see, e.g., [5] and [6]). As a starting point for our investigations we have chosen the form (1.1), (1.2). The systems (1.1), (1.2) and (1.3), (1.4) are also special cases of the integral-algebraic equations

$$
a(y)=a\left(y_{0}\right)+\int_{0}^{t} k(t, s, y(s)) d s
$$

considered in [2], where it is asssumed that $A=a_{y}=\partial a / \partial y$ is singular. In analogy with the theory of differential-algebraic equations (see, e.g., [4]) we call the systems (1.1), (1.2) and (1.3), (1.4) semi-explicit index 1 problems.

The system (1.1), (1.2) (and similarly (1.3), (1.4)) can be seen as the limit as $\varepsilon \rightarrow 0$ of the singularly perturbed problem

$$
\begin{gathered}
y(t)=f(t)+\int_{0}^{t}\left(K_{11}(t, s) y(s)+K_{12}(t, s) z(s)\right) d s, \\
\varepsilon z(t)=g(t)+\int_{0}^{t}\left(K_{21}(t, s) y(s)+K_{22}(t, s) z(s)\right) d s .
\end{gathered}
$$

Systems of the type (1.1), (1.2) also naturally arise when computing asymptotic expansions of the solution of (1.6), (1.7) and when studying numerical methods for such problems (see [7]).

The outline of this paper follows. In Section 2 we apply polynomial spline collocation methods to (1.1), (1.2) and (1.3), (1.4). A global convergence analysis is presented in Section 3, and Section 4 deals with superconvergence results. We conclude with a numerical illustration in Section 5.

\section{Polynomial Spline Collocation}

Let $\Pi_{N}$ be a uniform partition of the interval $I$ with grid points $t_{n}=n h$, $n=0, \ldots, N$, and let $h$ be the stepsize. Define the subintervals $\sigma_{0}=\left[t_{0}, t_{1}\right]$, $\sigma_{n}=\left(t_{n}, t_{n+1}\right], n=1, \ldots, N-1$. Let the collocation parameters be $0<c_{1}<$ $c_{2}<\cdots<c_{m} \leq 1$ and the collocation points be $t_{n j}=t_{n}+c_{j} h, j=1, \ldots, m$, $n=0, \ldots, N-1$. We consider polynomial spline approximations $u(t), v(t)$ of the exact solution $y(t), z(t)$ in the spline space

$$
S_{m-1}^{(-1)}\left(\Pi_{N}\right)=\left\{u: u_{n}=\left.u\right|_{\sigma_{n}} \in \pi_{m-1}, n=0, \ldots, N-1\right\} .
$$

This is the space of piecewise polynomials of degree (at most) $m-1$. Its dimension is $N m$, i.e., the same as the number of collocation points. In what follows we will consider the linear system (1.1), (1.2), but everything carries over to the nonlinear case (see Remark 4.2). We seek $u$ and $v$ such that the collocation equations

$$
\begin{array}{r}
u(t)=f(t)+\int_{0}^{t}\left(K_{11}(t, s) u(s)+K_{12}(t, s) v(s)\right) d s, \\
0=g(t)+\int_{0}^{t}\left(K_{21}(t, s) u(s)+K_{22}(t, s) v(s)\right) d s,
\end{array}
$$


are satisfied for $t=t_{n j}, j=1, \ldots, m, n=0, \ldots, N-1$. Let $Y_{n j}=u_{n}\left(t_{n j}\right)$ and $Z_{n j}=v_{n}\left(t_{n j}\right)$. Since $u_{n}, v_{n} \in \pi_{m-1}$, it holds for $\tau \in(0,1]$,

$$
u_{n}\left(t_{n}+\tau h\right)=\sum_{j=1}^{m} L_{j}(\tau) Y_{n j}, \quad v_{n}\left(t_{n}+\tau h\right)=\sum_{j=1}^{m} L_{j}(\tau) Z_{n j} .
$$

Here $L_{j}(\tau)=\Pi_{k \neq j}\left(\tau-c_{k}\right) /\left(c_{j}-c_{k}\right), j=1, \ldots, m$, denote the fundamental Lagrange polynomials. Inserting (2.3) into (2.1), (2.2), we obtain, for each $n=$ $0, \ldots, N-1$, a linear system for the unknowns $Y_{n j}, Z_{n j}, j=1, \ldots, m$,

$$
\begin{aligned}
Y_{n j}=f\left(t_{n j}\right) & +h \sum_{k=1}^{m}\left(\int_{0}^{c_{j}} K_{11}\left(t_{n j}, t_{n}+\tau h\right) L_{k}(\tau) d \tau\right) Y_{n k} \\
& +h \sum_{k=1}^{m}\left(\int_{0}^{c_{j}} K_{12}\left(t_{n j}, t_{n}+\tau h\right) L_{k}(\tau) d \tau\right) Z_{n k} \\
& +h \sum_{i=0}^{n-1} \sum_{k=1}^{m}\left(\int_{0}^{1} K_{11}\left(t_{n j}, t_{i}+\tau h\right) L_{k}(\tau) d \tau\right) Y_{i k} \\
& +h \sum_{i=0}^{n-1} \sum_{k=1}^{m}\left(\int_{0}^{1} K_{12}\left(t_{n j}, t_{i}+\tau h\right) L_{k}(\tau) d \tau\right) Z_{i k}, \\
0=g\left(t_{n j}\right) & +h \sum_{k=1}^{m}\left(\int_{0}^{c_{j}} K_{21}\left(t_{n j}, t_{n}+\tau h\right) L_{k}(\tau) d \tau\right) Y_{n k} \\
& +h \sum_{k=1}^{m}\left(\int_{0}^{c_{j}} K_{22}\left(t_{n j}, t_{n}+\tau h\right) L_{k}(\tau) d \tau\right) Z_{n k} \\
& +h \sum_{i=0}^{n-1} \sum_{k=1}^{m}\left(\int_{0}^{1} K_{21}\left(t_{n j}, t_{i}+\tau h\right) L_{k}(\tau) d \tau\right) Y_{i k} \\
& +h \sum_{i=0}^{n-1} \sum_{k=1}^{m}\left(\int_{0}^{1} K_{22}\left(t_{n j}, t_{i}+\tau h\right) L_{k}(\tau) d \tau\right) Z_{i k} .
\end{aligned}
$$

For the nonlinear system (1.3), (1.4) we obtain of course a nonlinear system for $Y_{n j}, Z_{n j}, j=1, \ldots, m$. In the gridpoints, the numerical solutions are defined as

$$
\begin{array}{rlrl}
y_{0} & =f\left(t_{0}\right), \\
y_{n+1} & =u_{n}\left(t_{n+1}\right), \quad \sum_{j=1}^{m} L_{j}(0) Z_{0 j}, & & \\
z_{n+1} & =v_{n}\left(t_{n+1}\right), \quad n=0, \ldots, N-1 .
\end{array}
$$

For the numerical solution of these systems, the integrals in (2.4), (2.5) have to be approximated by appropriate quadrature rules. Here we will use

$$
\begin{aligned}
\int_{0}^{c_{i}} f(\tau) d \tau & \approx \sum_{j=1}^{m} a_{i j} f\left(c_{j}\right), \quad i=1, \ldots, m, \\
\int_{0}^{1} f(\tau) d \tau & \approx \sum_{j=1}^{m} b_{j} f\left(c_{j}\right),
\end{aligned}
$$

where the coefficients $a_{i j}$ and $b_{j}$ are defined by $a_{i j}=\int_{0}^{c_{i}} L_{j}(\tau) d \tau, b_{j}=\int_{0}^{1} L_{j}(\tau) d \tau$, $i, j=1, \ldots, m$. The resulting approximations of $y, z$ are denoted by $\widehat{u}, \widehat{v}$ and are 
defined by

$$
\begin{aligned}
\widehat{Y}_{n j}=f\left(t_{n j}\right) & +h \sum_{k=1}^{m} a_{j k} K_{11}\left(t_{n j}, t_{n k}\right) \widehat{Y}_{n k}+h \sum_{k=1}^{m} a_{j k} K_{12}\left(t_{n j}, t_{n k}\right) \widehat{Z}_{n k} \\
& +h \sum_{i=0}^{n-1} \sum_{k=1}^{m} b_{k} K_{11}\left(t_{n j}, t_{i k}\right) \widehat{Y}_{i k}+h \sum_{i=0}^{n-1} \sum_{k=1}^{m} b_{k} K_{12}\left(t_{n j}, t_{i k}\right) \widehat{Z}_{i k}, \\
0=g\left(t_{n j}\right) & +h \sum_{k=1}^{m} a_{j k} K_{21}\left(t_{n j}, t_{n k}\right) \widehat{Y}_{n k}+h \sum_{k=1}^{m} a_{j k} K_{22}\left(t_{n j}, t_{n k}\right) \widehat{Z}_{n k} \\
& +h \sum_{i=0}^{n-1} \sum_{k=1}^{m} b_{k} K_{21}\left(t_{n j}, t_{i k}\right) \widehat{Y}_{i k}+h \sum_{i=0}^{n-1} \sum_{k=1}^{m} b_{k} K_{22}\left(t_{n j}, t_{i k}\right) \widehat{Z}_{i k},
\end{aligned}
$$

and equations similar to (2.3),$(2.6)$ and (2.7). The internal stages $\widehat{Y}_{n j}$ and $\widehat{Z}_{n j}$ of this discretized collocation method are thus equivalent to those of an extended implicit Volterra-Runge-Kutta method (see [1]).

\section{Global CONVERGence anAlysis}

Let $\|\cdot\|_{\infty}$ be the supremum norm. We have the following global convergence result:

Theorem 3.1. Consider the polynomial spline approximations $u, v$ in $S_{m-1}^{(-1)}\left(\Pi_{N}\right)$ to the solution $y, z$ of the system (1.1), (1.2) and defined by (2.3)-(2.5). For every choice of $c_{m},\left(0<c_{m} \leq 1\right)$, the collocation approximation $u$ converges to the solution $y$. If $c_{m}=1$, the collocation approximation $v$ converges to the solution $z$, and if $c_{m}<1$, the collocation approximation $v$ converges to the solution $z$ for any $m \geq 1$ if and only if

$$
-1 \leq R(\infty)=(-1)^{m} \prod_{i=1}^{m} \frac{1-c_{i}}{c_{i}} \leq 1 .
$$

Moreover, the following error estimates hold:

$$
\begin{aligned}
& \|y-u\|_{\infty}=O\left(h^{m}\right), \\
& \|z-v\|_{\infty}= \begin{cases}O\left(h^{m}\right) & \text { if } c_{m}=1, \\
O\left(h^{m}\right) & \text { if } c_{m}<1 \text { and }-1 \leq R(\infty)<1, \\
O\left(h^{m-1}\right) & \text { if } c_{m}<1 \text { and } R(\infty)=1,\end{cases}
\end{aligned}
$$

as $h \rightarrow 0$ with $N h \leq$ const.

Proof. It holds for the exact solution that

$$
\begin{array}{ll}
y\left(t_{n}+\tau h\right)=\sum_{j=1}^{m} L_{j}(\tau) y\left(t_{n j}\right)+r_{n}(\tau), & r_{n}(\tau)=h^{m} \frac{y^{(m)}\left(\eta_{n}(\tau)\right)}{m !} \prod_{i=1}^{m}\left(\tau-c_{i}\right), \\
z\left(t_{n}+\tau h\right)=\sum_{j=1}^{m} L_{j}(\tau) z\left(t_{n j}\right)+s_{n}(\tau), & s_{n}(\tau)=h^{m} \frac{z^{(m)}\left(\zeta_{n}(\tau)\right)}{m !} \prod_{i=1}^{m}\left(\tau-c_{i}\right),
\end{array}
$$


with $\tau \in[0,1]$. It follows that the errors $e=y-u$ and $\varepsilon=z-v$ have the following representation

$$
\begin{aligned}
& e_{n}\left(t_{n}+\tau h\right)=\sum_{j=1}^{m} L_{j}(\tau) e_{n}\left(t_{n j}\right)+r_{n}(\tau), \\
& \varepsilon_{n}\left(t_{n}+\tau h\right)=\sum_{j=1}^{m} L_{j}(\tau) \varepsilon_{n}\left(t_{n j}\right)+s_{n}(\tau),
\end{aligned}
$$

where $e_{n}=\left.e\right|_{\sigma_{n}}$ and $\varepsilon_{n}=\left.\varepsilon\right|_{\sigma_{n}}$. On the other hand, the errors satisfy the system

$$
\begin{aligned}
e_{n}\left(t_{n j}\right) & =\int_{0}^{t_{n j}}\left(K_{11}\left(t_{n j}, s\right) e(s)+K_{12}\left(t_{n j}, s\right) \varepsilon(s)\right) d s, \\
0 & =\int_{0}^{t_{n j}}\left(K_{21}\left(t_{n j}, s\right) e(s)+K_{22}\left(t_{n j}, s\right) \varepsilon(s)\right) d s .
\end{aligned}
$$

This gives

$$
\begin{aligned}
e_{n}\left(t_{n j}\right)= & h \int_{0}^{c_{j}}\left(K_{11}\left(t_{n j}, t_{n}+\tau h\right) e_{n}\left(t_{n}+\tau h\right)+K_{12}\left(t_{n j}, t_{n}+\tau h\right) \varepsilon_{n}\left(t_{n}+\tau h\right)\right) d \tau \\
& +h \sum_{i=0}^{n-1} \int_{0}^{1}\left(K_{11}\left(t_{n j}, t_{i}+\tau h\right) e_{i}\left(t_{i}+\tau h\right)+K_{12}\left(t_{n j}, t_{i}+\tau h\right) \varepsilon_{i}\left(t_{i}+\tau h\right)\right) d \tau,
\end{aligned}
$$

$$
\begin{aligned}
0= & h \int_{0}^{c_{j}}\left(K_{21}\left(t_{n j}, t_{n}+\tau h\right) e_{n}\left(t_{n}+\tau h\right)+K_{22}\left(t_{n j}, t_{n}+\tau h\right) \varepsilon_{n}\left(t_{n}+\tau h\right)\right) d \tau \\
& +h \sum_{i=0}^{n-1} \int_{0}^{1}\left(K_{21}\left(t_{n j}, t_{i}+\tau h\right) e_{i}\left(t_{i}+\tau h\right)+K_{22}\left(t_{n j}, t_{i}+\tau h\right) \varepsilon_{i}\left(t_{i}+\tau h\right)\right) d \tau .
\end{aligned}
$$

We now rewrite (3.7) with $n$ replaced by $n-1$ and $j=m$, subtract this equation from (3.7) and divide by $h$. (This process is the numerical analog of transforming a first kind equation into a second kind equation by differentation.) We obtain

$$
\begin{aligned}
\int_{0}^{c_{j}}\left(K_{21}\left(t_{n j}, t_{n}+\tau h\right) e_{n}\left(t_{n}+\tau h\right)+K_{22}\left(t_{n j}, t_{n}+\tau h\right) \varepsilon_{n}\left(t_{n}+\tau h\right)\right) d \tau \\
=\int_{0}^{c_{m}}\left(K_{21}\left(t_{n-1 m}, t_{n-1}+\tau h\right) e_{n-1}\left(t_{n-1}+\tau h\right)\right. \\
\left.\quad+K_{22}\left(t_{n-1 m}, t_{n-1}+\tau h\right) \varepsilon_{n-1}\left(t_{n-1}+\tau h\right)\right) d \tau \\
\quad-\sum_{i=0}^{n-1} \int_{0}^{1}\left(K_{21}\left(t_{n j}, t_{i}+\tau h\right) e_{i}\left(t_{i}+\tau h\right)+K_{22}\left(t_{n j}, t_{i}+\tau h\right) \varepsilon_{i}\left(t_{i}+\tau h\right)\right) d \tau \\
+\sum_{i=0}^{n-2} \int_{0}^{1}\left(K_{21}\left(t_{n-1 m}, t_{i}+\tau h\right) e_{i}\left(t_{i}+\tau h\right)\right. \\
\left.\quad+K_{22}\left(t_{n-1 m}, t_{i}+\tau h\right) \varepsilon_{i}\left(t_{i}+\tau h\right)\right) d \tau .
\end{aligned}
$$

We now have to distinguish between two cases, $c_{m}=1$ and $c_{m}<1$. 
Case I: $c_{m}=1$. Now (3.8) becomes

$$
\begin{aligned}
& \int_{0}^{c_{j}}\left(K_{21}\left(t_{n j}, t_{n}+\tau h\right) e_{n}\left(t_{n}+\tau h\right)+K_{22}\left(t_{n j}, t_{n}+\tau h\right) \varepsilon_{n}\left(t_{n}+\tau h\right)\right) d \tau \\
& =\sum_{i=0}^{n-1} \int_{0}^{1}\left(K_{21}\left(t_{n-1 m}, t_{i}+\tau h\right) e_{i}\left(t_{i}+\tau h\right)+K_{22}\left(t_{n-1 m}, t_{i}+\tau h\right) \varepsilon_{i}\left(t_{i}+\tau h\right)\right) d \tau \\
& \quad-\sum_{i=0}^{n-1} \int_{0}^{1}\left(K_{21}\left(t_{n j}, t_{i}+\tau h\right) e_{i}\left(t_{i}+\tau h\right)+K_{22}\left(t_{n j}, t_{i}+\tau h\right) \varepsilon_{i}\left(t_{i}+\tau h\right)\right) d \tau
\end{aligned}
$$

Using

$$
K_{k \ell}\left(t_{n-1 m}, t_{i}+\tau h\right)-K_{k \ell}\left(t_{n j}, t_{i}+\tau h\right)=h \partial_{1} K_{k l}\left(\cdot, t_{i}+\tau h\right), \quad k, \ell=1,2,
$$

with $\cdot$ between $t_{n-1 m}$ and $t_{n j}$,

$$
\int_{0}^{c_{j}} K_{22}\left(t_{n j}, t_{n}+\tau h\right) L_{k}(\tau) d \tau=K_{22}\left(t_{n}, t_{n}\right) a_{j k}+O(h), \quad j, k=1, \ldots, m,
$$

and with (3.2) and $(3.3)$, we arrive at

$$
\begin{aligned}
\sum_{k=1}^{m}\left(\int_{0}^{c_{j}} K_{21}\left(t_{n j}, t_{n}+\tau h\right) L_{k}(\tau) d \tau\right) e_{n}\left(t_{n k}\right) \\
\quad+\sum_{k=1}^{m}\left(K_{22}\left(t_{n}, t_{n}\right) a_{j k}+O(h)\right) \varepsilon_{n}\left(t_{n k}\right) \\
=h \sum_{i=0}^{n-1} \sum_{k=1}^{m}\left(\int_{0}^{1} \partial_{1} K_{21}\left(\cdot, t_{i}+\tau h\right) L_{k}(\tau) d \tau\right) e_{i}\left(t_{i k}\right) \\
\quad+h \sum_{i=0}^{n-1} \sum_{k=1}^{m}\left(\int_{0}^{1} \partial_{1} K_{22}\left(\cdot, t_{i}+\tau h\right) L_{k}(\tau) d \tau\right) \varepsilon_{i}\left(t_{i k}\right)+O\left(h^{m}\right)
\end{aligned}
$$

We also rewrite (3.6) using (3.2) and

$$
\begin{aligned}
e_{n}\left(t_{n j}\right)= & h \sum_{k=1}^{m}\left(\int_{0}^{c_{j}} K_{11}\left(t_{n j}, t_{n}+\tau h\right) L_{k}(\tau) d \tau\right) e_{n}\left(t_{n k}\right) \\
& +h \sum_{k=1}^{m}\left(\int_{0}^{c_{j}} K_{12}\left(t_{n j}, t_{n}+\tau h\right) L_{k}(\tau) d \tau\right) \varepsilon_{n}\left(t_{n k}\right) \\
& +h \sum_{i=0}^{n-1} \sum_{k=1}^{m}\left(\int_{0}^{1} K_{11}\left(t_{n j}, t_{i}+\tau h\right) L_{k}(\tau) d \tau\right) e_{i}\left(t_{i k}\right) \\
& +h \sum_{i=0}^{n-1} \sum_{k=1}^{m}\left(\int_{0}^{1} K_{12}\left(t_{n j}, t_{i}+\tau h\right) L_{k}(\tau) d \tau\right) \varepsilon_{i}\left(t_{i k}\right)+O\left(h^{m}\right)
\end{aligned}
$$


Let $E_{n}=\left(e_{n}\left(t_{n 1}\right), \ldots, e_{n}\left(t_{n m}\right)\right)^{\top}$ and $\epsilon_{n}=\left(\varepsilon_{n}\left(t_{n 1}\right), \ldots, \varepsilon_{n}\left(t_{n m}\right)\right)^{\top}, n=0, \ldots$, $N-1$. Then the previous two equations can be rewritten in matrix notation

$$
\begin{array}{r}
\left(\begin{array}{cc}
I-h K_{11}^{(n, n)} & -h K_{12}^{(n, n)} \\
K_{21}^{(n, n)} & K_{22}\left(t_{n}, t_{n}\right) A+O(h)
\end{array}\right)\left(\begin{array}{c}
E_{n} \\
\epsilon_{n}
\end{array}\right) \\
=h \sum_{i=0}^{n-1}\left(\begin{array}{cc}
K_{11}^{(n, i)} & K_{12}^{(n, i)} \\
\widetilde{K}_{21}^{(n, i)} & \widetilde{K}_{22}^{(n, i)}
\end{array}\right)\left(\begin{array}{c}
E_{i} \\
\epsilon_{i}
\end{array}\right)+O\left(h^{m}\right),
\end{array}
$$

where the meaning of the matrices $K_{k \ell}^{(n, i)}$ and $\widetilde{K}_{k \ell}^{(n, i)}$ is clear. Since $\left|K_{22}(t, t)\right| \geq$ $k_{0}>0$ for all $t \in I$, the inverse of the matrix on the left hand side exists and is bounded if $h$ is sufficiently small. It then follows from Gronwall's inequality that

$$
\left\|E_{n}\right\|=\max _{j=1, \ldots, m}\left\{\left|e_{n}\left(t_{n j}\right)\right|\right\}=O\left(h^{m}\right), \quad\left\|\epsilon_{n}\right\|=\max _{j=1, \ldots, m}\left\{\left|\varepsilon_{n}\left(t_{n j}\right)\right|\right\}=O\left(h^{m}\right),
$$

and the result follows from (3.2) and (3.3).

Case II: $c_{m}<1$. Using (3.8) and (3.10) we arrive at

$$
\begin{aligned}
& \int_{0}^{c_{j}}\left(K_{21}\left(t_{n j}, t_{n}+\tau h\right) e_{n}\left(t_{n}+\tau h\right)+K_{22}\left(t_{n j}, t_{n}+\tau h\right) \varepsilon_{n}\left(t_{n}+\tau h\right)\right) d \tau \\
& =\int_{0}^{c_{m}}\left(K_{21}\left(t_{n-1 m}, t_{n-1}+\tau h\right) e_{n-1}\left(t_{n-1}+\tau h\right)\right. \\
& \left.\quad+K_{22}\left(t_{n-1 m}, t_{n-1}+\tau h\right) \varepsilon_{n-1}\left(t_{n-1}+\tau h\right)\right) d \tau \\
& \quad-\int_{0}^{1}\left(K_{21}\left(t_{n j}, t_{n-1}+\tau h\right) e_{n-1}\left(t_{n-1}+\tau h\right)\right. \\
& \left.\quad+K_{22}\left(t_{n j}, t_{n-1}+\tau h\right) \varepsilon_{n-1}\left(t_{n-1}+\tau h\right)\right) d \tau \\
& +h \sum_{i=0}^{n-2} \int_{0}^{1}\left(\partial_{1} K_{21}\left(\cdot, t_{i}+\tau h\right) e_{i}\left(t_{i}+\tau h\right)+\partial_{1} K_{22}\left(\cdot, t_{i}+\tau h\right) \varepsilon_{i}\left(t_{i}+\tau h\right)\right) d \tau
\end{aligned}
$$

We now proceed as in the previous case. We insert (3.2) and (3.3), use 3.11),

$$
\int_{0}^{1} K_{22}\left(t_{n j}, t_{n-1}+\tau h\right) L_{k}(\tau) d \tau=K_{22}\left(t_{n-1}, t_{n-1}\right) b_{k}+O(h), \quad j, k=1, \ldots, m
$$

and similar expressions for $K_{21}$, divide by $K_{22}\left(t_{n}, t_{n}\right)$ and use

$$
\frac{K_{22}\left(t_{n-1}, t_{n-1}\right)}{K_{22}\left(t_{n}, t_{n}\right)}=1+O(h)
$$


We thus obtain

$$
\begin{aligned}
& \sum_{k=1}^{m}\left(\int_{0}^{c_{j}} \frac{K_{21}\left(t_{n j}, t_{n}+\tau h\right)}{K_{22}\left(t_{n}, t_{n}\right)} L_{k}(\tau) d \tau\right) e_{n}\left(t_{n k}\right) \\
&+\sum_{k=1}^{m}\left(a_{j k}+O(h)\right) \varepsilon_{n}\left(t_{n k}\right) \\
&= \sum_{k=1}^{m}\left(\frac{K_{21}\left(t_{n-1}, t_{n-1}\right)}{K_{22}\left(t_{n}, t_{n}\right)} a_{m k}+O(h)\right) e_{n-1}\left(t_{n-1 k}\right) \\
&+\sum_{k=1}^{m}\left(a_{m k}+O(h)\right) \varepsilon_{n-1}\left(t_{n-1 k}\right) \\
&-\sum_{k=1}^{m}\left(\frac{K_{21}\left(t_{n-1}, t_{n-1}\right)}{K_{22}\left(t_{n}, t_{n}\right)} b_{k}+O(h)\right) e_{n-1}\left(t_{n-1 k}\right) \\
&-\sum_{k=1}^{m}\left(b_{k}+O(h)\right) \varepsilon_{n-1}\left(t_{n-1 k}\right) \\
&+h \sum_{i=0}^{n-2} \sum_{k=1}^{m}\left(\int_{0}^{1} \frac{\partial_{1} K_{21}\left(\cdot, t_{i}+\tau h\right)}{K_{22}\left(t_{n}, t_{n}\right)} L_{k}(\tau) d \tau\right) e_{i}\left(t_{i k}\right) \\
&+h \sum_{i=0}^{n-2} \sum_{k=1}^{m}\left(\int_{0}^{1} \frac{\partial_{1} K_{22}\left(\cdot, t_{i}+\tau h\right)}{K_{22}\left(t_{n}, t_{n}\right)} L_{k}(\tau) d \tau\right) \varepsilon_{i}\left(t_{i k}\right)+O\left(h^{m}\right) .
\end{aligned}
$$

Together with (3.12) this gives in matrix notation

$$
\begin{aligned}
& \left(\begin{array}{cc}
I-h K_{11}^{(n, n)} & -h K_{12}^{(n, n)} \\
\widehat{K}_{21}^{(n, n)} & A+O(h)
\end{array}\right)\left(\begin{array}{c}
E_{n} \\
\epsilon_{n}
\end{array}\right) \\
& =\left(\begin{array}{cc}
h K_{11}^{(n, n-1)} & h K_{12}^{(n, n-1)} \\
\frac{K_{21}\left(t_{n-1}, t_{n-1}\right)}{K_{22}\left(t_{n}, t_{n}\right)} M_{1}+O(h) & M_{1}+O(h)
\end{array}\right)\left(\begin{array}{c}
E_{n-1} \\
\epsilon_{n-1}
\end{array}\right) \\
& \quad+h \sum_{i=0}^{n-2}\left(\begin{array}{ll}
K_{11}^{(n, i)} & K_{12}^{(n, i)} \\
\bar{K}_{21}^{(n, i)} & \bar{K}_{22}^{(n, i)}
\end{array}\right)\left(\begin{array}{c}
E_{i} \\
\epsilon_{i}
\end{array}\right)+O\left(h^{m}\right),
\end{aligned}
$$

where $M_{1}=\mathbb{1}_{m} e_{m}^{\top} A-\mathbb{1}_{m} b^{\top}$ and $\mathbb{1}_{m}=(1, \ldots, 1)^{\top}, e_{m}=(0, \ldots, 0,1)^{\top} \in \mathbb{R}^{m}$. It holds that the inverse of the matrix on the left hand side has the form

$$
\left(\begin{array}{cc}
I-h K_{11}^{(n, n)} & -h K_{12}^{(n, n)} \\
\widehat{K}_{21}^{(n, n)} & A+O(h)
\end{array}\right)^{-1}=\left(\begin{array}{cc}
I+O(h) & O(h) \\
\widehat{\widehat{K}}_{21}^{(n, n)} & A^{-1}+O(h)
\end{array}\right)
$$

if $h$ is sufficiently small and we have

$$
\begin{aligned}
&\left(\begin{array}{cc}
I-h K_{11}^{(n, n)} & -h K_{12}^{(n, n)} \\
\widehat{K}_{21}^{(n, n)} & A+O(h)
\end{array}\right)^{-1}\left(\begin{array}{cc}
h K_{11}^{(n, n-1)} & h K_{12}^{(n, n-1)} \\
\frac{K_{21}\left(t_{n-1}, t_{n-1}\right)}{K_{22}\left(t_{n}, t_{n}\right)} M_{1}+O(h) & M_{1}+O(h)
\end{array}\right) \\
&=\left(\begin{array}{cc}
O(h) & O(h) \\
\frac{K_{21}\left(t_{n-1}, t_{n-1}\right)}{K_{22}\left(t_{n}, t_{n}\right)} M_{0}+O(h) & M_{0}+O(h)
\end{array}\right)
\end{aligned}
$$


where $M_{0}=A^{-1} M_{1}=A^{-1} \mathbb{1}_{m}\left(e_{m}^{\top} A-b^{\top}\right)$. Thus (3.14) becomes

$$
\left(\begin{array}{c}
E_{n} \\
\epsilon_{n}
\end{array}\right)=\left(\begin{array}{cc}
0 & 0 \\
\frac{K_{21}\left(t_{n-1}, t_{n-1}\right)}{K_{22}\left(t_{n}, t_{n}\right)} M_{0} & M_{0}
\end{array}\right)\left(\begin{array}{c}
E_{n-1} \\
\epsilon_{n-1}
\end{array}\right)+h \sum_{i=0}^{n-1}\left(\begin{array}{ll}
M_{2} & M_{3} \\
M_{4} & M_{5}
\end{array}\right)\left(\begin{array}{c}
E_{i} \\
\epsilon_{i}
\end{array}\right)+O\left(h^{m}\right),
$$

where $M_{i}, i=2, \ldots, 5$, denote bounded matrices. Since the matrix multiplying $\left(E_{n-1}, \epsilon_{n-1}\right)^{\top}$ is diagonalizable, we can conclude as in [9] with the help of the following lemma and Lemma 6 of [3].

Lemma 3.1. Let $M_{0}$ be defined as above. Then $M_{0}$ has rank one and its only nonzero eigenvalue is

$$
R(\infty)=(-1)^{m} \prod_{i=1}^{m} \frac{1-c_{i}}{c_{i}}
$$

where $R(z)=1+z b^{\top}(I-z A)^{-1} \mathbb{1}_{m}$ denotes the stability function of the Runge-Kutta method $(c, A, b)$.

Proof of Lemma 3.1. $M_{0}$ has rank one because $\mathbb{1}_{m}\left(e_{m}^{\top} A-b^{\top}\right)$ has rank one. Therefore the only nonzero eigenvalue of $M_{0}$ is equal to its trace. Let $A^{-1}=\left(\omega_{i j}\right)_{i, j=1}^{m}$. Then

$$
\operatorname{tr}\left(M_{0}\right)=\sum_{i=1}^{m}\left(a_{m i}-b_{i}\right) \sum_{j=1}^{m} \omega_{i j}=1-b^{\top} A^{-1} \mathbb{1}_{m}=R(\infty) .
$$

The result of the lemma now follows from Theorem IV.3.10 of [4].

Remark 3.1. Using standard techniques it can be shown that the results of Theorem 3.1 also hold for the discretized collocation approximations $\widehat{u}$ and $\widehat{v}$.

\section{Superconvergence Results}

In this section we will show that for an adequate choice of the collocation parameters $c_{j}$, the order of convergence for $u$ is higher than $m$ in the gridpoints $t_{n}$ (superconvergence). Since for Volterra equations of the first kind superconvergence does in general not occur (see [1]), we cannot expect a higher order of convergence for $v$ in the gridpoints.

Let the residuals $\delta_{i}, i=1,2$, be defined by

$$
\begin{aligned}
u(t) & =f(t)-\delta_{1}(t)+\int_{0}^{t}\left(K_{11}(t, s) u(s)+K_{12}(t, s) v(s)\right) d s, \\
0 & =g(t)-\delta_{2}(t)+\int_{0}^{t}\left(K_{21}(t, s) u(s)+K_{22}(t, s) v(s)\right) d s,
\end{aligned}
$$

for all $t \in I$. It follows from (2.1), 2.2) that $\delta_{i}(t)=0, i=1,2$, if $t=t_{n j}$, i.e., the residuals vanish in the collocation points. Moreover the residuals $\delta_{i}$ are smooth on each subinterval $\sigma_{n}$ and $\delta_{2}$ is continuous on $I$.

The collocation errors now verify for $t \in I$,

$$
\begin{aligned}
e(t) & =\delta_{1}(t)+\int_{0}^{t}\left(K_{11}(t, s) e(s)+K_{12}(t, s) \varepsilon(s)\right) d s, \\
0 & =\delta_{2}(t)+\int_{0}^{t}\left(K_{21}(t, s) e(s)+K_{22}(t, s) \varepsilon(s)\right) d s .
\end{aligned}
$$


Our aim is to write $e$ in terms of the residuals $\delta_{1}$ and $\delta_{2}$. Differentiating (4.4) and using resolvent representations for $\varepsilon$ and $e$, one easily shows that there exist sufficiently smooth functions $L(t, s)$ and $M(t, s)$ such that

$$
e(t)=\delta_{1}(t)+\int_{0}^{t} L(t, s) \delta_{1}(s) d s+\int_{0}^{t} M(t, s) \delta_{2}^{\prime}(s) d s, \quad t \in I .
$$

Here $\delta_{2}^{\prime}$ denotes the right derivative of $\delta_{2}$. Hence for $t=t_{n}$, we have with integration by parts

$$
\begin{aligned}
e\left(t_{n}\right)= & \delta_{1}\left(t_{n}\right)+\sum_{i=0}^{n-1} \int_{t_{i}}^{t_{i+1}}\left(L\left(t_{n}, s\right) \delta_{1}(s)+M\left(t_{n}, s\right) \delta_{2}^{\prime}(s)\right) d s \\
= & \delta_{1}\left(t_{n}\right)+\sum_{i=0}^{n-1}\left(M\left(t_{n}, t_{i+1}\right) \delta_{2}\left(t_{i+1}\right)-M\left(t_{n}, t_{i}\right) \delta_{2}\left(t_{i}\right)\right. \\
& \left.\quad+\int_{t_{i}}^{t_{i+1}}\left(L\left(t_{n}, s\right) \delta_{1}(s)-\partial_{2} M\left(t_{n}, s\right) \delta_{2}(s)\right) d s\right) .
\end{aligned}
$$

We proceed as for Volterra equations of the second kind (see [1]). The integrals in (4.7) are approximated by quadrature formulas based on (2.9). Since the residuals vanish in the collocation points, these integrals are equal to the quadrature errors $E_{n i}^{(1)}$ and $E_{n i}^{(2)}$. It holds that

$$
e\left(t_{n}\right)=\delta_{1}\left(t_{n}\right)+M\left(t_{n}, t_{n}\right) \delta_{2}\left(t_{n}\right)+h \sum_{i=0}^{n-1}\left(E_{n i}^{(1)}+E_{n i}^{(2)}\right) .
$$

If $c_{m}=1$, we have $\delta_{i}\left(t_{n}\right)=0, i=1,2$, and the error $e\left(t_{n}\right)$ is equal to the quadrature error induced by the formula (2.9). Therefore superconvergence can be obtained for a suitable choice of the collocation parameters $c_{j}$. For $c_{m}<1$, we have in general that $\delta_{i}\left(t_{n}\right) \neq 0, i=1,2$, (compare [1]) and we do not have superconvergence. We have thus showed the following theorem.

Theorem 4.1. If the collocation parameters $c_{j}$ are the Radau II points for $(0,1]$ (i.e., the zeros of $P_{m-1}(2 s-1)-P_{m}(2 s-1)$ where $P_{m}$ denotes the Legendre polynomial of degree $m$ ), then

$$
\max \left\{\left|y\left(t_{n}\right)-u\left(t_{n}\right)\right|: n=0, \ldots, N\right\}=O\left(h^{2 m-1}\right),
$$

as $h \rightarrow 0$ with $N h \leq$ const.

Remark 4.1. It is known that superconvergence does not occur for the collocation approximation to the solution of a Volterra equation of the second kind if the $c_{j}$ are the Gauss points (cf. Theorem 5.3.3(c) of [1]). However the full order of superconvergence can be recovered for the iterated collocation approximation. For the system of integral-algebraic equations (1.1), (1.2) considered in this paper, we do not obtain the full order of superconvergence for the iterated collocation approximation. More precisely let $u, v \in S_{m-1}^{(-1)}\left(Z_{N}\right)$ be the polynomial spline approximations to $y, z$ as defined in Section 2. We define the iterated collocation approximation $u^{I}$ by

$$
u^{I}(t)=f(t)+\int_{0}^{t}\left(K_{11}(t, s) u(s)+K_{22}(t, s) v(s)\right) d s, \quad t \in I .
$$


Together with (4.1) it follows that

$$
u^{I}(t)-u(t)=\delta_{1}(t), \quad t \in I,
$$

i.e., the iterated collocation approximation $u^{I}$ and the collocation approximation $u$ coincide in the collocation points $t_{n j}$. Iterated collocation is therefore only interesting for methods with $c_{m}<1$. Let $e^{I}=y-u^{I}$ be the iterated error. In view of (4.10) and (4.5) we have that

$$
e^{I}(t)=e(t)-\delta_{1}(t)=\int_{0}^{t} L(t, s) \delta_{1}(s) d s+\int_{0}^{t} M(t, s) \delta_{2}^{\prime}(s) d s, \quad t \in I .
$$

We continue as we did before for $e$. However due to the presence of $\delta_{2}\left(t_{n}\right)$ (cf. (4.8)), we cannot expect high order superconvergence in the case where $c_{m}<1$. Nevertheless it is interesting to note that numerical evidence shows that for Gauss methods $\left|y\left(t_{n}\right)-u^{I}\left(t_{n}\right)\right|$ is $O\left(h^{m+1}\right)$ if $m$ is odd and $O\left(h^{m}\right)$ if $m$ is even.

Remark 4.2. Using standard techniques, one shows that all previous convergence results also hold for the nonlinear system (1.3), (1.4).

Remark 4.3. In case of a pure equation of the second kind, (i.e., (1.1) without the $z$-component and without (1.2)), the result of Theorem 3.1 reduces to the result of Theorem 5.3.2 of [1]: one observes global convergence of order $m$ for $y$. Theorem 4.1 reduces to the result of Theorem 5.3.3(a) of [1: for Radau II collocation parameters one has superconvergence of order $2 m-1$ for $y$ at the gridpoints. Moreover one has local superconvergence for the iterated collocation approximation to $y$ (Radau II and Gauss parameters).

In case of a pure equation of the first kind (i.e., (1.2) without the $y$-component and without (1.1) ), the result of Theorem 3.1 can be compared to the result of Theorem 5.5.1a of [1]. Moreover, no local superconvergence for $z$ can be achieved (cf. Theorem 5.5.2(b) of [1]).

The convergence estimates of Theorems 3.1 and 4.1 are similar to those for the convergence of Runge-Kutta methods for semi-explicit differential-algebraic equations of index 1: except for stiffly accurate methods, one generally observes an order reduction for the $z$-component (for details see, e.g., Theorem VI.1.1 of [4]).

\section{Numerical illustration}

We solved (1.3), (1.4) on $[0,1]$ with $k(t, s, y, z)=\exp (t-s) y^{2} z, \ell(t, s, y, z)=$ $(1+t-s) y z$ and $f, g$ such that the exact solution is $y(t)=\exp (-t), z(t)=\cos (t)$. We collocated at the Radau II points with $m=1, \ldots, 4$ and $N=4,8,16,32,64$. We computed the observed orders of convergence from the maximum errors $e$ and $\varepsilon$ at the gridpoints. The results are listed in Tables 1 and 2 and they nicely confirm Theorems 3.1 and 4.1 .

TABLE 1. Orders of convergence for $u$

\begin{tabular}{|c|c|c|c|c|}
\hline$N$ & 8 & 16 & 32 & 64 \\
\hline$m=1$ & 1.13 & 1.06 & 1.03 & 1.01 \\
\hline$m=2$ & 2.99 & 3.00 & 3.00 & 3.00 \\
\hline$m=3$ & 4.96 & 4.99 & 5.00 & 5.00 \\
\hline$m=4$ & 6.99 & 7.00 & 7.00 & 7.00 \\
\hline
\end{tabular}


TABLE 2. Orders of convergence for $v$

\begin{tabular}{|c|c|c|c|c|}
\hline$N$ & 8 & 16 & 32 & 64 \\
\hline$m=1$ & 1.94 & 1.31 & 1.13 & 1.06 \\
\hline$m=2$ & 2.10 & 2.04 & 2.02 & 2.01 \\
\hline$m=3$ & 3.11 & 3.05 & 3.03 & 3.01 \\
\hline$m=4$ & 4.11 & 4.00 & 3.98 & 3.99 \\
\hline
\end{tabular}

Remark 5.1. A comparison of spline collocation and pseudospectral methods for integral-algebraic equations (1.3), (1.4) can be found in [8].

\section{ACKNOWLEDGMENT}

This research was supported by the Swiss National Science Foundation (Research Fellowship 8220-042835).

\section{REFERENCES}

[1] H. Brunner and P. J. van der Houwen. The Numerical Solution of Volterra Equations, volume 3 of CWI Monographs. North Holland, Amsterdam, 1986. MR 88g:65136

[2] C. W. Gear. Differential algebraic equations, indices, and integral algebraic equations. SIAM J. Numer. Anal., 27:1527-1534, 1990. MR 91m:65192

[3] E. Hairer, Ch. Lubich, and S. P. Nørsett. Order of convergence of one-step methods for Volterra integral equations of the second kind. SIAM J. Numer. Anal., 20:569-579, 1983. MR 84g:65163

[4] E. Hairer and G. Wanner. Solving Ordinary Differential Equations II. Stiff and DifferentialAlgebraic Problems. Second Revised Edition. Springer-Verlag, Berlin - Heidelberg, 1996. MR 97m:65007

[5] J. Janno and L. von Wolfersdorf. Identification of weakly singular memory kernels in heat conduction. Z. Angew. Math. Mech. 77:243-257, 1997. MR 98d:35233

[6] J. Janno and L. von Wolfersdorf. Inverse problems for identification of memory kernels in viscoelasticity. Technical Report 96-04, Fakultät für Mathematik und Informatik, Technische Universität Bergakademie Freiberg, 1996.

[7] J.-P. Kauthen. A survey of singularly perturbed Volterra equations. Appl. Num. Math., 24:95114, 1997. MR 98e:45003

[8] J.-P. Kauthen. The numerical solution of Volterra integral-algebraic equations by collocation methods. In A. Sydow, editor, 15th IMACS World Congress on Scientific Computation, Modelling and Applied Mathematic, Berlin, August 1997, Volume 2: Numerical Mathematics, pages 451-456, Berlin, 1997. Wissenschaft und Technik Verlag.

[9] J.-P. Kauthen and H. Brunner. Continuous collocation approximations to solutions of first kind Volterra equations. Math. Comp., 66:1441-1459, 1997. MR 98f:65128

Dufourstrasse 23, CH-3005 Bern, Switzerland

E-mail address: kauthen@bluewin.ch 